\title{
THE NUMBER OF SINGULAR FIBERS IN HYPERELLIPTIC LEFSCHETZ FIBRATIONS
}

\author{
TÜLIN ALTUNÖZ
}

\begin{abstract}
We consider complex surfaces, viewed as smooth 4-dimensional manifolds, that admit hyperelliptic Lefschetz fibrations over the 2-sphere. In this paper, we show that the minimal number of singular fibers of such fibrations is equal to $2 g+4$ for even $g \geq 4$. For odd $g \geq 7$, we show that the number is greater than or equal to $2 g+6$. Moreover, we discuss the minimal number of singular fibers in all hyperelliptic Lefschetz fibrations over the 2-sphere as well.
\end{abstract}

\section{INTRODUCTION}

Donaldson and Gompf's results ([9], [10], [12] and [13]) give the relation between symplectic 4-manifolds and Lefschetz fibrations, which are a fibering of a 4-manifold by surfaces, with a finite number of singularities of a prescribed type. Donaldson proved that every symplectic 4-manifold admits a Lefschetz pencil, which can be blown up at its base points to obtain a Lefschetz fibration. On the other hand, Gompf proved that any 4-manifold admitting a Lefschetz fibration carries a symplectic structure. The isomorphism class of a Lefschetz fibration is determined by its global monodromy. This relation provides a combinatorial way to understand any symplectic 4-manifold via its monodromy, whenever it exists.

A Lefschetz fibration admits certain singular fibers associated to its monodromy. The results on the number of singular fibers of a Lefschetz fibration give us important information about the total space. It is well known that the number of singular fibers in a Lefschetz fibration cannot be arbitrary. A natural question to ask is what the minimal number of singular fibers in Lefschetz fibrations is.

Let $M_{g, h}$ denote the minimal number of singular fibers in all nontrivial relatively minimal Lefschetz fibrations of fiber genus $g$ and base genus $h$. Even though the exact value of $M_{g, h}$ for $h \geq 1$ is almost known (except the numbers $M_{g, 1}$ for $g \geq 3$ and $\left.M_{2,2}\right)[14,18,19,25,30]$, this question is still open when $h=0$ and $g \geq 3$. It was proved that $M_{g, 0} \leq 2 g+4$ when $g$ is even and $M_{g, 0} \leq 2 g+10$ when $g$ is odd $[7,8,16]$. It is known that $M_{2,0}=7$ by Xiao's construction [32]. Recently, a relation among seven positive Dehn twists in the mapping class group of genus-2 surface was found by Baykur and Korkmaz [4]. They also constructed an interesting relation consisting of 12 positive Dehn twists along simple closed curves which are invariant under a hyperelliptic involution $\iota$ in the mapping class group of genus-3 surface. Moreover, they showed that the number of singular fibers in all genus-3 hyperelliptic Lefschetz fibrations over the 2 -sphere is greater than or equal to 12 .

2000 Mathematics Subject Classification: 57M99, 20F38

Keywords: Mapping class groups, Lefschetz fibrations 
Let $N_{g}$ denote the minimal number of singular fibers in all genus- $g$ hyperelliptic Lefschetz fibrations over the 2 -sphere having at least one singular fiber. It follows from the result of Baykur and Korkmaz that $N_{3}=12$. For $g \geq 4$, it is known that $N_{g} \leq 2 g+4$ (respectively $N_{g} \leq 8 g+4$ ) when $g$ is even (respectively when $g$ is odd). (Here, $8 g+4$ comes from the hyperelliptic relation.)

Let $M_{g}$ denote the minimal number of singular fibers in all genus- $g$ hyperelliptic Lefschetz fibrations on a complex surface over the 2-sphere having at least one singular fiber. Here, by a complex surface we mean a compact connected complex analytic manifold of complex dimension 2, considered as a smooth 4-dimensional manifold.

Our aim in this paper is to estimate the numbers $N_{g}$ and $M_{g}$ for $g \geq 4$. For the number $M_{g}$, we have the following results:

Theorem 1.1. For all even $g \geq 4, M_{g}=2 g+4$.

Theorem 1.2. For all odd $g \geq 7, M_{g} \geq 2 g+6$.

For the number $N_{g}$ with $4 \leq g \leq 10$, we have the following results:

Theorem 1.3. For the number $N_{g}$ the following holds.

(1) $N_{4}=12$,

(2) $N_{5} \geq 15$,

(3) $N_{6}=16$,

(4) $N_{7} \geq 17$

(5) $N_{8}=19$ or 20 ,

(6) $N_{9} \geq 24$,

(7) $N_{10}=23$ or 24 .

Here is an outline of the paper. In Section 2, we give some relevant background information from the theory of Lefschetz fibrations and some results to be used in the sequel. Section 3 investigates the minimal number of singular fibers in hyperelliptic Lefschetz fibrations on complex surfaces. In this section, we prove Theorems 1.1 and 1.2. In Section 4, we investigate the minimal number of singular fibers in hyperelliptic Lefschetz fibrations. We examine these numbers for $4 \leq g \leq$ 10 and prove Theorem 1.3.

Acknowledgements. I would like to thank my advisor Mustafa Korkmaz for many invaluable comments and discussions. Thanks are due to Anar Akhmedov and T.J. Li for helpful conversations. I also thank the referee and the editor for reading the paper very carefully, making many valuable suggestions and corrections. This paper is a part of the author's Ph.D. thesis [1] at Middle East Technical University. The author was partially supported by the Scientific and Technological Research Council of Turkey (TÜBİTAK).

\section{PRELIMINARIES}

We start with a review of some basic definitions and properties of Lefschetz fibrations. In this paper, we denote the 2 -sphere by $\mathbb{S}^{2}$. Let $\Sigma_{g}$ denote a closed connected oriented surface of genus- $g$ and $\operatorname{Mod}_{g}$ denote the mapping class group of $\Sigma_{g}$, i.e., the group of isotopy classes of orientation-preserving diffeomorphisms of $\Sigma_{g}$. Let $M$ be a closed connected oriented smooth 4-dimensional manifold. A smooth surjective map $f: M \rightarrow \mathbb{S}^{2}$ is a Lefschetz fibration with connected oriented 
genus- $g$ regular fiber if it has finitely many critical points and around each critical point it is written in the form of $f\left(z_{1}, z_{2}\right)=z_{1}^{2}+z_{2}^{2}$ with respect to some local complex coordinates agreeing with the orientations of $M$ and $\mathbb{S}^{2}$. The genus- $g$ of a regular fiber $F$ is called the genus of the fibration. We assume that all the critical points lie in distinct fibers, called singular fibers, which can be obtained after a small perturbation. Each singular fiber is obtained by shrinking a simple closed curve, called vanishing cycle, in the regular fiber. If the vanishing cycle is nonseparating (respectively separating), then the singular fiber is said to be irreducible (respectively reducible). In this paper, we also assume that all Lefschetz fibrations are nontrivial, i.e. it has at least one singular fiber and fibrations are relatively minimal, i.e. it has no fiber containing a sphere of self-intersection -1 , otherwise one can blow-down it without changing the rest of the fibration.

Lefschetz fibrations can be described combinatorially by their monodromy representations. The monodromy of a Lefschetz fibration $f: M \rightarrow \mathbb{S}^{2}$ is given by a positive factorization $t_{\alpha_{1}} t_{\alpha_{2}} \ldots t_{\alpha_{n}}=1$ in $\operatorname{Mod}_{g}$, where $\alpha_{i}$ are the vanishing cycles of the singular fibers. (Here $t_{a}$ denotes the positive Dehn twist about a simple closed curve $a$ on a genus- $g$ surface.) Conversely, for a given positive factorization $t_{a_{1}} t_{a_{2}} \ldots t_{a_{k}}=1$ in $\operatorname{Mod}_{g}$, one can construct a genus- $g$ Lefschetz fibration over $\mathbb{S}^{2}$ by attaching 2 -handles along vanishing cycles $a_{i}$ in a $\Sigma_{g}$ fiber in $\Sigma_{g} \times D^{2}$ with -1 framing, and then by closing it up by a fiber preserving map to get a fibration over $\mathbb{S}^{2}$. Two Lefschetz fibrations $f_{1}: M_{1} \rightarrow \mathbb{S}^{2}$ and $f_{2}: M_{2} \rightarrow \mathbb{S}^{2}$ are said to be isomorphic if there exist orientation preserving diffeomorphisms $H: M_{1} \rightarrow M_{2}$ and $h: \mathbb{S}^{2} \rightarrow \mathbb{S}^{2}$ such that $f_{2} H=h f_{1}$. If $g \geq 2$, it is known that a genus- $g$ Lefschetz fibration over $\mathbb{S}^{2}$ is characterized by a positive factorization of the identity element in $\operatorname{Mod}_{g}$ up to Hurwitz moves (exchanging subwords $t_{a_{i}} t_{a_{i+1}}=t_{a_{i+1}} t_{t_{a_{i+1}}\left(a_{i}\right)}$ ) and global conjugations (changing each $t_{a_{i}}$ with $t_{\varphi\left(a_{i}\right)}$ for some $\varphi \in \operatorname{Mod}_{g}$ ).

The hyperelliptic mapping class group $\operatorname{HMod}_{g}$ of $\Sigma_{g}$ is defined to be the subgroup of the mapping class group $\operatorname{Mod}_{g}$ which is the centralizer of the class of a hyperelliptic involution $\iota: \Sigma_{g} \rightarrow \Sigma_{g}$. We say that a genus- $g$ Lefschetz fibration is hyperelliptic if its vanishing cycles are invariant under the hyperelliptic involution $\iota$ up to isotopy.

We collect some useful facts about the first homology group of the hyperelliptic mapping class group.

Recall that for any group $G$, the first homology group of $G$ with integral coefficient is the abelianization of $G$, that is,

$$
H_{1}(G ; \mathbb{Z})=G /[G, G]
$$

where $[G, G]$ is the subgroup of $G$ generated by all commutators $[a, b]=a b a^{-1} b^{-1}$ for all $a, b \in G$. It is known that $H_{1}\left(\operatorname{Mod}_{g} ; \mathbb{Z}\right)$ is a cyclic group generated by the class of a Dehn twist about a non-separating simple closed curve and also we have the following lemma:

Lemma 2.1. For a closed orientable surface of genus $g \geq 1$, we have the following isomorphism of the first homology group $H_{1}\left(\operatorname{Mod}_{g} ; \mathbb{Z}\right)$ of the mapping class group $\operatorname{Mod}_{g}$ :

$$
H_{1}\left(\operatorname{Mod}_{g} ; \mathbb{Z}\right) \cong \begin{cases}\mathbb{Z}_{12}, & \text { if } g=1, \\ \mathbb{Z}_{10}, & \text { if } g=2, \\ 0, & \text { if } g \geq 3\end{cases}
$$


For the proof of Lemma 2.1 and further details about the homology groups of the mapping class group, see [17].

The following lemma can be proven by the presentation of the hyperelliptic mapping class group [5].

Lemma 2.2. For a closed orientable surface of genus $g \geq 1$, the first homology group $H_{1}\left(\operatorname{HMod}_{g} ; \mathbb{Z}\right)$ of the hyperelliptic mapping class group $\operatorname{HMod}_{g}$ has the following isomorphism:

$$
H_{1}\left(\operatorname{HMod}_{g} ; \mathbb{Z}\right) \cong \begin{cases}\mathbb{Z} / 4(2 g+1), & \text { if } g \text { is odd }, \\ \mathbb{Z} / 2(2 g+1), & \text { if } g \text { is even } .\end{cases}
$$

All Dehn twists about non-separating simple closed curves that are invariant under the hyperelliptic involution $\iota$ on $\Sigma_{g}$ are nontrivial in the hyperelliptic mapping class group of $\Sigma_{g}, \operatorname{HMod}_{g}$, and each of them maps to the same generator in $H_{1}\left(\operatorname{HMod}_{g} ; \mathbb{Z}\right)$ under the natural $\operatorname{map} \operatorname{HMod}_{g} \rightarrow H_{1}\left(\operatorname{HMod}_{g} ; \mathbb{Z}\right)$. If a product of positive Dehn twists about non-separating curves in $\operatorname{HMod}_{g}$ is trivial then the number of twists is divisible by $4(2 g+1)$ (respectively $2(2 g+1)$ ) when $g$ is odd (respectively even). A separating simple closed curve on $\Sigma_{g}$ is said to be of type $h$ if it bounds subsurfaces of genera $h$ and $g-h$. By the even chain relation, each positive Dehn twist about a separating simple closed curve of type $h$ can be written as a product of $2 h(4 h+2)$ positive Dehn twists about non-separating simple closed curves. This implies the following relation between the number of non-separating singular fibers and that of separating singular fibers in a genus- $g$ hyperelliptic Lefschetz fibration:

Lemma 2.3. Let $n$ (or $s$ ) be the number of non-separating (resp. separating) vanishing cycles in a genus-g hyperelliptic Lefschetz fibration over $\mathbb{S}^{2}$. Then, we have

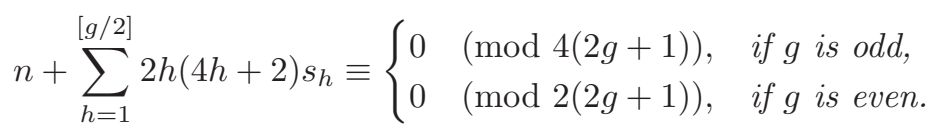

where $s=\sum_{h=1}^{[g / 2]} s_{h}$, and $s_{h}$ is the number of separating vanishing cycles of type $h$.

Lemma 2.4. [11, 23, 24] Let $f: X \rightarrow \mathbb{S}^{2}$ be a genus-g hyperelliptic Lefschetz fibration. Let $n$ and $s=\sum_{h=1}^{[g / 2]} s_{h}$ be the numbers of non-separating and separating vanishing cycles of this fibration, respectively, where $s_{h}$ denotes the number of separating vanishing cycles that separate the genus-g surface into two surfaces one of which has genus $h$. Then the signature of $X$ is

$$
\sigma(X)=-\frac{g+1}{2 g+1} n+\sum_{h=1}^{[g / 2]}\left(\frac{4 h(g-h)}{2 g+1}-1\right) s_{h} .
$$

Remark 2.5. Ozbagci [26] concluded that $\sigma(X) \leq n-s$ for any 4-manifold $X$ admitting a genus- $g$ Lefschetz fibration over $\mathbb{S}^{2}$ or $\mathbb{D}^{2}$ and he also proved that

$$
\sigma(X) \leq n-s-4
$$

when the Lefschetz fibration over $\mathbb{S}^{2}$ is hyperelliptic. It can be easily obtained that $\sigma(X) \leq n-s-2$ using $b_{1}(X) \leq 2 g-1$ by the handlebody decomposition of nontrivial Lefschetz fibrations over $\mathbb{S}^{2}$ and the fact that every nontrivial Lefschetz 
fibration over $\mathbb{S}^{2}$ has at least one non-separating vanishing cycle. Then, Cadavid [7] improved the upper bound of signature $\sigma(X)$, showing that

$$
\sigma(X) \leq n-s-2\left(2 g-b_{1}(X)\right) .
$$

Let us recall the following Stipsicz's theorem, which we will use to examine the number of singular fibers.

Theorem 2.6. [29] Let $f: X \rightarrow \mathbb{S}^{2}$ be a nontrivial genus-g Lefschetz fibration with $b_{2}^{+}(X)=1$.

(1) If $g \geq 6$ is even, then $f: X \rightarrow \mathbb{S}^{2}$ admits at least $2 g+4$ singular fibers. (This lower bound is sharp.)

(2) If $g \geq 15$ is odd, then $f: X \rightarrow \mathbb{S}^{2}$ admits at least $2 g+10$ singular fibers. (This lower bound is sharp.)

(3) If $g \geq 9$ is odd, then $f: X \rightarrow \mathbb{S}^{2}$ contains at least $2 g+6$ singular fibers.

We want to remark that in the above theorem the lower bounds in (1) and (2) are sharp, that is, the minimum values $2 g+4$ and $2 g+10$, respectively, can be realized on ruled surfaces which are uniquely determined as $\left(\Sigma_{g / 2} \times \mathbb{S}^{2}\right) \# 4 \overline{\mathbb{C} P^{2}}$ and $\left(\Sigma_{(g-1) / 2} \times \mathbb{S}^{2}\right) \# 8 \overline{\mathbb{C} P^{2}}$, respectively [29, Sections 4.1 and 4.2$]$. However, in (3), the lower bound may not be sharp, that is, we do not know whether there exists a Lefschetz fibration $f: X \rightarrow \mathbb{S}^{2}$ with $b_{2}^{+}(X)=1$ and $2 g+6$ singular fibers.

\section{The Minimal NUMBer of SingUlar FIBERS IN HYPERELLIPTIC LEFSCHETZ FIBRATIONS ON COMPLEX SURFACES}

3.1. Even genus case. In this section, first we prove some lemmas to prove Theorem 1.1.

Lemma 3.1. The 4-manifold $\left(\Sigma_{2} \times S^{2}\right) \# 3 \overline{\mathbb{C} P^{2}}$ does not admit a genus-4 Lefschetz fibration over $\mathbb{S}^{2}$.

Proof. Suppose that $\left(\Sigma_{2} \times \mathbb{S}^{2}\right) \# 3 \overline{\mathbb{C} P^{2}}$ admits a genus-4 Lefschetz fibration and consider the homology class of a regular fiber $F$. We may write

$$
[F]=a[U]+b[V]+\sum_{i=1}^{3} c_{i}\left[E_{i}\right] \in H_{2}\left(\left(\Sigma_{2} \times \mathbb{S}^{2}\right) \# 3 \overline{\mathbb{C} P^{2}} ; \mathbb{Z}\right),
$$

for some integers $a, b$ and $c_{i}$, where $[U],[V]$ denote the homology classes of the section and fiber of the ruling $\Sigma_{2} \times S^{2} \rightarrow \Sigma_{2}$, respectively, such that $[U]^{2}=[V]^{2}=0$, $[U] \cdot[V]=1$, and $\left[E_{i}\right]$ denote the homology class of the exceptional sphere of the ith blow-up.

The composition of the blowing down and the projection map $\Sigma_{2} \times \mathbb{S}^{2} \rightarrow \Sigma_{2}$ leads to a degree- $d$ map $F \rightarrow \Sigma_{2}$ for some integer $d$. The degree $d$ must be equal to $a$. Moreover, since the fiber of the trivial $\mathbb{S}^{2}$-bundle $\Sigma_{2} \times \mathbb{S}^{2} \rightarrow \Sigma_{2}$ has a pseudoholomorphic representative [21], the degree of the map $F \rightarrow \Sigma_{2}$ is positive by the positivity of intersection.

Consider a singular fiber $\Sigma$. Since the normalization of $\Sigma$ has genus $\leq 3$, such a degree- $d$ map yields the following inequality

$$
3-1 \geq g(\Sigma)-1 \geq d(2-1)=a(2-1),
$$


where $g(\Sigma)$ is the genus of the fiber $\Sigma[15]$. Therefore, $0<d=a \leq 2$. Since $[F]^{2}=0$, we have

$$
2 a b=\sum_{i=1}^{3} c_{i}^{2} .
$$

Since the symplectic structure on $\left(\Sigma_{2} \times \mathbb{S}^{2}\right) \# 3 \overline{\mathbb{C} P^{2}}$ is unique up to deformations and diffeomorphisms, we can apply the adjunction formula

$$
2 g(F)-2=[F]^{2}+[K] \cdot[F],
$$

where $[K]=-2[U]+(2 h-2)[V]+\left[E_{1}\right]+\left[E_{2}\right]+\left[E_{3}\right]$ is the canonical class with $h=g\left(\Sigma_{2}\right)=2$. In this case, the adjunction formula gives

$$
2 g(F)-2=2 a h-2 a-2 b-\sum_{i=1}^{3} c_{i} .
$$

Thus, for $g(F)=4$ and $h=2$, we have

$$
6=2 a-2 b-\sum_{i=1}^{3} c_{i} .
$$

For $a=1$, by the identities (3.1) and (3.3) we have

$$
\sum_{i=1}^{3} c_{i}^{2}=2 b \text { and } \sum_{i=1}^{3} c_{i}=-4-2 b
$$

which lead to

$$
\sum_{i=1}^{3} c_{i}^{2}+\sum_{i=1}^{3} c_{i}=-4
$$

Hence $\sum_{i=1}^{3}\left(c_{i}+\frac{1}{2}\right)^{2}=-\frac{13}{4}$, which is not possible.

In the case $a=2$, using the identities (3.1) and (3.3), we have the following equalities:

$$
4 b=\sum_{i=1}^{3} c_{i}^{2} \text { and } 2=-2-\sum_{i=1}^{3} c_{i},
$$

which give

$$
\sum_{i=1}^{3} c_{i}^{2}+2 \sum_{i=1}^{3} c_{i}=-4 .
$$

Thus, the resulting equality is $\sum_{i=1}^{3}\left(c_{i}+1\right)^{2}=-1$, which is a contradiction. Therefore, this shows that $\left(\Sigma_{2} \times \mathbb{S}^{2}\right) \# 3 \overline{\mathbb{C} P^{2}}$ does not admit a genus-4 Lefschetz fibration over $\mathbb{S}^{2}$.

Remark 3.2. The proof of the above theorem is based on Stipsicz's technique in [29, Lemma 4.4], and some arguments of [6, Theorem 21] and [20, Lemma 4.2]. It implies that Theorem 2.6 (1) is true for $g=4$ and similarly, one can also show that Theorem 2.6 (3) holds for $g=7$. 
Let $e(X)$ denote the Euler characteristic of a 4-manifold $X$. For a genus- $g$ Lefschetz fibration $f: M \rightarrow \mathbb{S}^{2}$ with $n$ separating and $s$ non-separating vanishing cycles, we have

$$
e(M)=4-4 g+n+s .
$$

We define the following two invariants associated to the 4-manifold $M$ :

$$
\chi_{h}(M)=\frac{e(M)+\sigma(M)}{4} \text { and } c_{1}^{2}(M)=2 e(X)+3 \sigma(X) .
$$

Note that if $M$ is a complex surface, then $\chi_{h}(M)$ is the holomorphic Euler characteristic of $M$ and $c_{1}^{2}(M)$ is the square of the first Chern class of $M$.

Lemma 3.3. Let $f$ be a genus-g hyperelliptic Lefschetz fibration on a complex surface $X$ over $\mathbb{S}^{2}$ with even $g \geq 6$ or odd $g \geq 9$. If $n+s<2 g+4$, then $n \geq 2 g+2$.

Proof. Suppose that there exists a hyperelliptic Lefschetz fibration on a complex surface $X$ with $n<2 g+2$.

Let us first consider $n<2 g$. Using the inequality $\sigma(X) \leq n-s-4$ for hyperelliptic Lefschetz fibrations over $\mathbb{S}^{2}[26]$, we have

$$
\begin{aligned}
\chi_{h}(X) & =\frac{e(X)+\sigma(X)}{4} \\
& \leq \frac{(4-4 g+n+s)+(n-s-4)}{4} \\
& =\frac{2 n-4 g}{4}<0 .
\end{aligned}
$$

Now, assume that $n=2 g$, which gives rise to $s \leq 3$. By the signature formula, we get

$$
\begin{aligned}
\sigma(X) & =-\frac{g+1}{2 g+1} n+\sum_{h=1}^{[g / 2]}\left(\frac{4 h(g-h)}{2 g+1}-1\right) s_{h} \\
& \leq-\frac{g+1}{2 g+1}(2 g)+3\left(\frac{4(g / 2)(g / 2)}{2 g+1}-1\right) \\
& =\frac{g^{2}-8 g-3}{2 g+1} \\
& <\frac{g}{2}-3
\end{aligned}
$$

and also, using $n+s \leq 2 g+3$ we have

$$
\begin{aligned}
\chi_{h}(X) & =\frac{e(X)+\sigma(X)}{4} \\
& <\frac{4-4 g+2 g+3+(g / 2)-3}{4} \\
& \leq \frac{-3(g / 2)+4}{4}<0 .
\end{aligned}
$$

Hence, we conclude that $\chi_{h}(X)<0$ if $n \leq 2 g$. By the classification of complex surfaces, $X$ is diffeomorphic to a blow up of a ruled surface which implies that $b_{2}^{+}=1[2]$. However, this contradicts Theorem 2.6. Therefore, $n>2 g$. Since the number $n$ is even by the equality in Lemma 2.3, we get the required inequality. 
Proof of Theorem 1.1. Suppose that we have a hyperelliptic Lefschetz fibration on a complex surface $X$ with $n+s<2 g+4$ and $g \geq 6$ is even. Hence, $n \geq 2 g+2$ by Lemma 3.3. The equality in Lemma 2.3 implies that $n$ is even and also $s=$ $\sum_{h=1}^{[g / 2]} s_{h}>0$. Thus, $n=2 g+2$ and $s=1$.

The signature $\sigma(X)$ of $X$ is computed using the signature formula as follows:

$$
\begin{aligned}
\sigma(X) & =-\frac{g+1}{2 g+1} n+\sum_{h=1}^{[g / 2]}\left(\frac{4 h(g-h)}{2 g+1}-1\right) s_{h} \\
& \leq-\frac{g+1}{2 g+1}(2 g+2)+\left(\frac{4(g / 2)(g / 2)}{2 g+1}-1\right) \\
& =-\frac{g^{2}+6 g+3}{2 g+1} \\
& <-\frac{g}{2} .
\end{aligned}
$$

Using $\sigma(X)<-\frac{g}{2}, n=2 g+2$ and $s=1$, we get:

$$
\begin{aligned}
\chi_{h}(X) & =\frac{e(X)+\sigma(X)}{4} \\
& <\frac{4-4 g+2 g+3-(g / 2)}{4} \\
& \leq \frac{-5(g / 2)+7}{4}<0 .
\end{aligned}
$$

In this case, the classification of complex surfaces implies that $X$ is a blow-up of a ruled surface and hence $b_{2}^{+}=1$. However, this is impossible if $g \geq 6$ by Theorem 2.6. Thus $M_{g} \geq 2 g+4$. For even $g \geq 6$, the existence of the genus- $g$ hyperelliptic Lefschetz fibration over $\mathbb{S}^{2}$ with $2 g+4$ singular fibers $[7,8,16]$ implies that $M_{g}=2 g+4$.

Now, consider the remaining case, $g=4$. Assume that there exists a hyperelliptic Lefschetz fibration so that $n+s<2 g+4=12$. The equation in Lemma 2.3 leads

\begin{tabular}{|c|c|c|c|c|}
\hline & $\left(n, s_{1}, s_{2}\right)$ & $e(X)$ & $\sigma(X)$ & $c_{1}^{2}(X)$ \\
\hline & $(6,1,0)$ & -5 & -3 & -19 \\
\hline & $(6,4$, & -2 & -2 & -10 \\
\hline & $(6,0$, & -3 & -1 & -9 \\
\hline & $(8,2,1)$ & -1 & -3 & -11 \\
\hline
\end{tabular}
to $n+12 s_{1}+4 s_{2} \equiv 0(\bmod 18)$, where $s=s_{1}+s_{2}$ and $n$ is even. Moreover, we have $n \geq 6$ using the inequality $n \geq \frac{1}{5}(8 g-3)=\frac{29}{5}$ given in [6].

The possible triples $\left(n, s_{1}, s_{2}\right)$ and some topological invariants of the corresponding genus-4 Lefschetz fibrations over $\mathbb{S}^{2}$, which can be easily computed using the signature formula and $e(X)=4-4 g+n+s=-12+n+s_{1}+s_{2}$, are given as follows:

We now rule out all cases:

Case (a1). In this case, $c_{1}^{2}(X)=-19<4-4 g=-12$, which gives a contradiction [28].

Cases (a2)-(a4). In these cases, $c_{1}^{2}(X)<2-2 g=-6$. This implies that $X$ is a blow-up of a rational or ruled surface [20]. Thus we have $b_{2}^{+}(X)=1$. Moreover, 
using inequality (2.1), one can conclude that $X$ cannot be simply-connected and so it is a blow-up of a ruled surface. The equalities

$$
\begin{aligned}
e(X) & =2-2 b_{1}(X)+b_{2}^{+}(X)+b_{2}^{-}(X) \\
& =3-2 b_{1}(X)+b_{2}^{-}(X)
\end{aligned}
$$

and

$$
\sigma(X)=b_{2}^{+}(X)-b_{2}^{-}(X)=1-b_{2}^{-}(X)
$$

imply that $b_{1}(X)=4$. Hence, $X$ is diffeomorphic to $\left(\Sigma_{2} \times S^{2}\right) \# m \overline{\mathbb{C} P^{2}}$. (Note that $m=2,1$ and 3 for the cases (a2), (a3) and (a4), respectively). From the proof of Lemma 3.1, we see that $\left(\Sigma_{2} \times S^{2}\right) \# m \overline{\mathbb{C} P^{2}}$ cannot admit a genus-4 Lefschetz fibration over $\mathbb{S}^{2}$ for $m=0,1,2,3$. Since there is a hyperelliptic genus-4 Lefschetz fibration with 12 singular fibers $[7,8,16]$, we have $M_{4}=12$. This proves our claim.

3.2. Odd genus case. In this section, we find a lower bound for the number $M_{g}$ when $g \geq 7$ is odd.

Proof of Theorem 1.2. Suppose that there exists a hyperelliptic Lefschetz fibration on a complex surface $X$ with odd $g \geq 7$ and $n+s<2 g+6$.

First consider the case $g \geq 9$. If $n<2 g$, then it can be shown that $\chi_{h}(X)<0$ using the inequality $\sigma(X) \leq n-s-4$ as in the proof of Lemma 3.3. This implies that $b_{2}^{+}=1$ by the classification of complex surfaces. But, this gives a contradiction with Theorem 2.6. The odd case of the equation in Lemma 2.3 leads to $n \equiv 0$ (mod 4 ). We can conclude that $n \geq 2 g+2$. The assumption $n+s<2 g+6$ gives rise to $n=2 g+2$ and $s \leq 3$. Therefore, the signature formula implies the following inequality:

$$
\begin{aligned}
\sigma(X) & =-\frac{g+1}{2 g+1} n+\sum_{h=1}^{[g / 2]}\left(\frac{4 h(g-h)}{2 g+1}-1\right) s_{h} \\
& \leq-\frac{g+1}{2 g+1}(2 g+2)+3\left(\frac{4(g / 2)(g / 2)}{2 g+1}-1\right) \\
& =\frac{g^{2}-10 g-5}{2 g+1} \\
& <\frac{g}{2}-5 .
\end{aligned}
$$

Then, using the inequality $\sigma(X)<\frac{g}{2}-5$, the holomorphic Euler characteristic satisfies

$$
\begin{aligned}
\chi_{h}(X) & =\frac{e(X)+\sigma(X)}{4}=\frac{4-4 g+n+s+\sigma(X)}{4} \\
& <\frac{4-4 g+2 g+5+(g / 2)-5}{4} \\
& \leq \frac{-3 g}{8}+1<0 .
\end{aligned}
$$

Hence, the classification of complex surfaces implies that $X$ is a blow-up of a ruled surface. In this case, $b_{2}^{+}(X)=1$. However, this contradicts to Theorem 2.6. 
Now consider the case $g=7$. Suppose that we have a hyperelliptic genus-7 Lefschetz fibration $X$ with $n+s<20$, where $s=s_{1}+s_{2}+s_{3}$. We know that $n \geq \frac{1}{5}(8 g-3)=\frac{53}{5}$ (and therefore $\left.n \geq 11\right)[6]$ and it follows from the congruence $n+12 s_{1}-20 s_{2}+24 s_{3} \equiv 0 \quad(\bmod 60)$

that $n \equiv 0(\bmod 4)$ by Lemma 2.3. Hence the possible values of $\left(n, s_{1}, s_{2}, s_{3}\right)$, $e(X), \sigma(X), c_{1}^{2}(X)$ and $\chi_{h}(X)$ are as follows:

\begin{tabular}{r|c|c|c|c|c|}
\multicolumn{1}{c}{$\left(n, s_{1}, s_{2}, s_{3}\right)$} & $e(X)$ & $\sigma(X)$ & $c_{1}^{2}(X)$ & $\chi_{h}(X)$ \\
\cline { 2 - 6 } (b1) & $(12,0,0,2)$ & -10 & -2 & -26 & -3 \\
\cline { 2 - 6 } (b2) & $(12,2,0,1)$ & -9 & -3 & -27 & -3 \\
\cline { 2 - 6 } (b3) & $(12,4,0,0)$ & -8 & -4 & -28 & -3 \\
\cline { 2 - 6 } (b4) & $(12,1,0,4)$ & -7 & 3 & -5 & -1 \\
\cline { 2 - 6 } (b5) & $(12,0,3,2)$ & -7 & 3 & -5 & -1 \\
\cline { 2 - 6 } (b6) & $(12,3,0,3)$ & -6 & 2 & -12 & -1 \\
\cline { 2 - 6 } (b7) & $(12,2,3,1)$ & -5 & 1 & -7 & -1 \\
\cline { 2 - 6 } (b8) & $(12,0,0,7)$ & -5 & 9 & -5 & 1 \\
\cline { 2 - 6 } (b9) & $(12,5,0,2)$ & -5 & 1 & -7 & -1 \\
\cline { 2 - 6 } (b10) & $(12,4,3,0)$ & -5 & 1 & -7 & -1 \\
\cline { 2 - 6 } (b11) & $(16,0,2,1)$ & -5 & -3 & -19 & -2 \\
\cline { 2 - 6 } & & & &
\end{tabular}

Cases (b1)-(b3). The manifold $X$ has $c_{1}^{2}(X)<4-4 g=-24$, which gives a contradiction [28].

Cases (b4)-(b7), (b9) and (b10). In these cases, $\chi_{h}(X)<0$. Thus, $X$ is a blow-up of a ruled surface. However, $\sigma(X) \leq 0$ for such a manifold. Hence, we exclude these cases.

Case (b8). In this case, the manifold $X$ does not satisfy the inequality $\sigma(X) \leq$ $n-s-4$.

Case (b11). In this case, since $c_{1}^{2}(X)<2-2 g=-12, X$ is diffeomorphic to a blow-up of a rational or ruled surface. Hence $b_{2}^{+}=1$. We have

$$
\begin{aligned}
e(X) & =-5=2-2 b_{1}(X)+b_{2}^{+}(X)+b_{2}^{-}(X) \\
& =3-2 b_{1}(X)+b_{2}^{-}(X)
\end{aligned}
$$

and

$$
\begin{aligned}
\sigma(X) & =-3=b_{2}^{+}(X)-b_{2}^{-}(X) \\
& =1-b_{2}^{-}(X) .
\end{aligned}
$$

Hence $\left(b_{1}(X), b_{2}^{+}(X), b_{2}^{-}(X)\right)=(6,1,4)$. Therefore, $X=\left(\Sigma_{3} \times S^{2}\right) \# 3 \overline{\mathbb{C} P^{2}}$. But one can prove that $\left(\Sigma_{3} \times S^{2}\right) \# 3 \overline{\mathbb{C} P^{2}}$ does not admit a genus-7 Lefschetz fibration over $\mathbb{S}^{2}$ using the same idea as in the proof of Lemma 3.1. This finishes the proof.

\section{The minimal number of Singular FiBers in HYPERELLIPTIC LEFSCHETZ FIBRATIONS}

In this section, we determine the minimal number of singular fibers in some hyperelliptic Lefschetz fibrations over $\mathbb{S}^{2}$. The proofs of Theorems 1.1 and 1.2 rely on the fact that any complex surface admitting a symplectic structure with $\chi_{h}<0$ is diffeomorphic to a ruled surface. In this section, we study the minimal number 
of singular fibers in hyperelliptic Lefschetz fibrations over $\mathbb{S}^{2}$ that may not have a complex structure. Recall that $N_{g}$ denotes the minimal number of singular fibers in all hyperelliptic genus- $g$ Lefschetz fibrations over $\mathbb{S}^{2}$.

Proof of Theorem 1.3. One can easily conclude that $N_{4}=12$ and $N_{7} \geq 17$ by the proofs of Theorems 1.1 and 1.2 (for $g=7$, refer to the cases (b1)-(b3)), respectively.

Now let us begin the proof of Theorem 1.3 (2). Suppose that $N_{5}<15$ so that we have a hyperelliptic genus-5 Lefschetz fibration $X$. Let $n$ and $s=s_{1}+s_{2}$ be the numbers of nonseparating and separating vanishing cycles, respectively. Hence $n+s<15$.

The equation in Lemma 2.3 turns out to be

$$
n+12 s_{1}-4 s_{2} \equiv 0 \quad(\bmod 44)
$$

so that $n$ is divided by 4 . It is known that $n \geq 8[6]$. The signature and the Euler characteristic are computed as

$$
\sigma(X)=\frac{-6 n+5 s_{1}+13 s_{2}}{11}
$$

and

$$
e(X)=4-4 g+n+s=-16+n+s_{1}+s_{2}
$$

respectively. Hence the possible values of $\left(n, s_{1}, s_{2}\right), e(X), \sigma(X), c_{1}^{2}(X)$ and $\chi_{h}(X)$ are as follows:

\begin{tabular}{c|c|c|c|c|c|} 
& $\left(n, s_{1}, s_{2}\right)$ & $e(X)$ & $\sigma(X)$ & $c_{1}^{2}(X)$ & \multicolumn{1}{c}{$\chi_{h}(X)$} \\
\cline { 2 - 6 }$(\mathrm{c} 1)$ & $(8,0,2)$ & -6 & -2 & -18 & -2 \\
\cline { 2 - 6 }$(\mathrm{c} 2)$ & $(8,3,0)$ & -5 & -3 & -19 & -2 \\
\cline { 2 - 6 }$(\mathrm{c} 3)$ & $(8,1,5)$ & -2 & 2 & 2 & 0 \\
\cline { 2 - 6 } & &
\end{tabular}

We now eliminate all cases:

Cases (c1) and (c2). In these cases, $c_{1}^{2}(X)<4-4 g=-16$. This is impossible [28].

Case (c3). In this case, $\sigma(X)>n-s-4$, which is also impossible for hyperelliptic Lefschetz fibrations [26]. Therefore, $N_{5}$ cannot be less than 15.

Next, we will prove that $N_{6}=16$. Suppose that $N_{6}<16$ so that we have a hyperelliptic genus-6 Lefschetz fibration $X$ with $n+s<16$, where $s=s_{1}+s_{2}+s_{3}$. Using arguments similar to the above, we have the possible values of $\left(n, s_{1}, s_{2}, s_{3}\right)$, $e(X), \sigma(X)$ and $c_{1}^{2}(X)$ are as follows:

\begin{tabular}{c|c|c|c|c|}
\multicolumn{1}{c}{$\left(n, s_{1}, s_{2}, s_{3}\right)$} & $e(X)$ & $\sigma(X)$ & $c_{1}^{2}(X)$ \\
\cline { 2 - 5 }$(\mathrm{d} 1)$ & $(10,0,3,0)$ & -7 & -1 & -17 \\
\cline { 2 - 5 }$(\mathrm{d} 2)$ & $(10,3,0,1)$ & -6 & -2 & -18 \\
\cline { 2 - 5 }$(\mathrm{d} 3)$ & $(10,2,0,3)$ & -5 & 1 & -7 \\
\cline { 2 - 5 }$(\mathrm{d} 4)$ & $(10,1,4,0)$ & -5 & 1 & -7 \\
\cline { 2 - 5 }$(\mathrm{d} 5)$ & $(12,0,1,0)$ & -7 & -5 & -29 \\
\cline { 2 - 5 } (d6) & $(12,1,2,0)$ & -5 & -3 & -19 \\
\cline { 2 - 5 } (d7) & $(14,1,0,0)$ & -5 & -7 & -31 \\
\cline { 2 - 5 } & & \multicolumn{3}{|c}{}
\end{tabular}

We now eliminate all cases:

Cases $(\mathrm{d} 5)$ and $(\mathrm{d} 7)$. In these cases, $c_{1}^{2}(X)<4-4 g=-22$. This is a contradiction $[28]$. 
Cases (d1), (d2) and (d6). In these cases, $c_{1}^{2}<2-2 g=-10$. Hence, $X$ is a blow-up of a rational or ruled surface [20]. Thus, $b_{2}^{+}(X)=1$. However, this contradicts to Theorem 2.6.

Cases (d3) and (d4). In these cases, we have the following identities:

$$
\begin{aligned}
\sigma(X) & =b_{2}^{+}(X)-b_{2}^{-}(X)=1 \\
e(X) & =2-2 b_{1}(X)+b_{2}^{+}(X)+b_{2}^{-}(X)=-5 .
\end{aligned}
$$

So, the equations (4.1) and (4.2) yield

$$
\begin{aligned}
& b_{2}^{+}(X)=b_{1}(X)-3 \\
& b_{2}^{-}(X)=b_{1}(X)-4 .
\end{aligned}
$$

Observe that $X$ cannot be a rational surface because $b_{1}(X)=4>0$ as $b_{2}^{+}=1$. Also, $X$ is not a blow-up of a ruled surface, since ruled surfaces have $\sigma \leq 0$. Let $\widetilde{X}$ be the minimal model of $X$ so that $X \cong \widetilde{X} \# k \overline{\mathbb{C P}}^{2}$ for some non-negative integer $k$. Due to Liu [22] and Taubes [31], $c_{1}^{2}(\widetilde{X}) \geq 0$. Also, the equation

$$
c_{1}^{2}(\widetilde{X})=c_{1}^{2}(X)+k=-7+k
$$

implies that $k \geq 7$. It is known that $b_{2}^{-}(X) \geq k \geq 7$. The identity (4.4) gives rise to $b_{1}(X) \geq 11$. Since $b_{1}(X) \leq 2 g-1=11$ by the theory of Lefcshetz fibrations, we have $b_{1}(X)=11$. However, this contradicts with the result of [20, Lemma 2.5]. Hence $N_{6}$ cannot be less than 16 . Since there exists a genus-6 hyperelliptic Lefschetz fibration with 16 singular fibers $[7,8,16]$, we have $N_{6}=16$.

For $8 \leq g \leq 10$, we list all possible values of the numbers $n$ and $s$ (the remaining details for these cases follow similarly from the above arguments). One can list these numbers using the congruence in Lemma 2.3 and the inequality $n \geq(8 g-3) / 5[6]$.

For $g=8$, the possible values of $\left(n, s_{1}, s_{2}, s_{3}, s_{4}\right), e(X), \sigma(X)$ and $c_{1}^{2}(X)$ are as follows:

\begin{tabular}{r|c|c|c|c|}
\multicolumn{1}{c}{$\left(n, s_{1}, s_{2}, s_{3}, s_{4}\right)$} & $e(X)$ & $\sigma(X)$ & $c_{1}^{2}(X)$ \\
\cline { 2 - 5 }$(\mathrm{e} 1)$ & $(14,1,0,0,1)$ & -12 & -4 & -36 \\
\cline { 2 - 5 }$(\mathrm{e} 2)$ & $(14,0,2,0,1)$ & -11 & -1 & -25 \\
\cline { 2 - 5 }$(\mathrm{e} 3)$ & $(14,0,1,3,0)$ & -10 & 2 & -14 \\
\cline { 2 - 5 }$(\mathrm{e} 4)$ & $(16,1,1,0,0)$ & -10 & -6 & -38 \\
\cline { 2 - 5 }$(\mathrm{e} 5)$ & $(14,0,1,2,2)$ & -9 & 5 & -3 \\
\cline { 2 - 5 } & & &
\end{tabular}

\begin{tabular}{|c|c|c|c|c|}
\hline & $\left(n, s_{1}, s_{2}, s_{3}, s_{4}\right)$ & $e(X)$ & $\sigma(X)$ & $c_{1}^{2}(X)$ \\
\hline (f1) & $(16,0,0,0,2)$ & -14 & -2 & -34 \\
\hline (f2) & $(16,1,1,1,0)$ & -13 & -3 & -35 \\
\hline$(\mathrm{t} 3$ & $(16,0,0,1,3)$ & -12 & 4 & -12 \\
\hline$(\mathrm{f} 4$ & $(16,5,0,0,0)$ & -11 & -5 & -37 \\
\hline$(\mathrm{f} 5$ & $(16,1,1,2,1)$ & -11 & 3 & -13 \\
\hline f6 & $(16,0,3,2,0)$ & -11 & 3 & -13 \\
\hline f7) & $(16,3,1,0,2)$ & -10 & 2 & -14 \\
\hline
\end{tabular}

Using arguments similar to those arguments, we can eliminate all possibilities except for the case (e5). Thus, we can conclude that $N_{8}=19$ or 20.

For $g=9$, the possible values of $\left(n, s_{1}, s_{2}, s_{3}, s_{4}\right), e(X), \sigma(X)$ and $c_{1}^{2}(X)$ are as follows: 
THE NUMBER OF SINGULAR FIBERS IN HYPERELLIPTIC LEFSCHETZ FIBRATIONS 13

\begin{tabular}{r|c|c|c|c|}
\multicolumn{1}{c}{} & \multicolumn{1}{c}{$\left(n, s_{1}, s_{2}, s_{3}, s_{4}\right)$} & $e(X)$ & $\sigma(X)$ & \multicolumn{1}{c}{$c_{1}^{2}(X)$} \\
\cline { 2 - 5 } (f8) & $(16,3,0,3,0)$ & -10 & 2 & -14 \\
\cline { 2 - 5 } (f9) & $(16,2,3,0,1)$ & -10 & 2 & -14 \\
\cline { 2 - 5 } (f10) & $(16,1,5,0,0)$ & -10 & 2 & -14 \\
\cline { 2 - 5 } (f11) & $(16,0,0,2,4)$ & -10 & 10 & 10 \\
\cline { 2 - 5 } (f12) & $(16,5,0,1,1)$ & -9 & 1 & -15 \\
\cline { 2 - 5 } (f13) & $(16,4,2,1,0)$ & -9 & 1 & -15 \\
\cline { 2 - 5 } (f14) & $(16,2,0,0,5)$ & -9 & 9 & 9 \\
\cline { 2 - 5 } (f15) & $(16,1,2,0,4)$ & -9 & 9 & 9 \\
(f16) & $(16,1,1,3,2)$ & -9 & 9 & 9 \\
\cline { 2 - 5 } (f17) & $(16,1,0,6,0)$ & -9 & 9 & 9 \\
(f18) & $(16,0,4,0,3)$ & -9 & 9 & 9 \\
\cline { 2 - 5 } (f19) & $(16,0,3,3,1)$ & -9 & 9 & 9 \\
\cline { 2 - 5 } (f20) & $(20,0,1,2,0)$ & -9 & -3 & -27 \\
\cline { 2 - 5 } & & &
\end{tabular}

Using arguments similar to those above, we can eliminate all possibilities (f1) - (f20) such that $n+s<24$. Thus, we can conclude that $N_{9} \geq 24$.

For $g=10$, the possible values of $\left(n, s_{1}, s_{2}, s_{3}, s_{4}, s_{5}\right), e(X), \sigma(X)$ and $c_{1}^{2}(X)$ are as follows:

\begin{tabular}{l|c|c|c|c|}
\multicolumn{1}{c}{} & $\left(n, s_{1}, s_{2}, s_{3}, s_{4}, s_{5}\right)$ & $e(X)$ & $\sigma(X)$ & \multicolumn{1}{c}{$c_{1}^{2}(X)$} \\
\cline { 2 - 5 } (g1) & $(16,0,1,0,1,1)$ & -17 & 1 & -31 \\
\cline { 2 - 5 } (g2) & $(16,1,2,0,1,0)$ & -16 & 0 & -32 \\
\cline { 2 - 5 } (g3) & $(16,0,1,1,1,1)$ & -16 & 4 & -20 \\
\cline { 2 - 5 } (g4) & $(16,1,2,1,1,0)$ & -15 & 3 & -21 \\
\cline { 2 - 5 } (g5) & $(16,1,0,0,2,2)$ & -15 & 7 & -9 \\
\cline { 2 - 5 } (g6) & $(16,0,2,0,0,3)$ & -15 & 7 & -9 \\
\cline { 2 - 5 } (g7) & $(16,0,1,2,1,1)$ & -15 & 7 & -9 \\
\cline { 2 - 5 } (g8) & $(16,4,0,0,0,2)$ & -14 & 2 & -22 \\
\cline { 2 - 5 } (g9) & $(16,2,1,0,2,1)$ & -14 & 6 & -10 \\
\cline { 2 - 5 } (g10) & $(16,1,3,0,0,2)$ & -14 & 6 & -10 \\
\cline { 2 - 5 } (g11) & $(16,1,2,2,1,0)$ & -14 & 6 & -10 \\
\cline { 2 - 5 } (g12) & $(16,1,0,1,2,2)$ & -14 & 10 & 2 \\
\cline { 2 - 5 } (g13) & $(16,0,2,1,0,3)$ & -14 & 10 & 2 \\
\cline { 2 - 5 } (g14) & $(16,0,2,0,4,0)$ & -14 & 10 & 2 \\
\cline { 2 - 5 } (g15) & $(16,0,0,0,1,5)$ & -14 & 14 & 14 \\
\cline { 2 - 5 } (g16) & $(18,2,0,0,0,0)$ & -16 & -8 & -56 \\
\cline { 2 - 5 } (g17) & $(18,2,0,1,0,0)$ & -15 & -5 & -45 \\
\cline { 2 - 5 } (g18) & $(18,2,0,2,0,0)$ & -14 & -2 & -34 \\
\cline { 2 - 5 } (g19) & $(18,1,0,0,3,0)$ & -14 & 2 & -22 \\
\cline { 2 - 5 } (g20) & $(18,0,2,0,0,1)$ & -14 & 2 & -22 \\
\cline { 2 - 5 } (g21) & $(20,1,0,0,0,1)$ & -14 & -6 & -46 \\
\cline { 2 - 5 } (g22) & $(18,0,0,0,2,3)$ & -13 & 9 & 1 \\
\cline { 2 - 5 } & & &
\end{tabular}

Using arguments similar to those above arguments, one can eliminate all possibilities except for the case (g22). Thus, one can conclude that $N_{10}=23$ or 24 .

As long as genus- $g$ increases, the number of possibilities of $n$ and $s$ increases, where $n$ and $s$ are the numbers of irreducible and reducible fibers, respectively. 
Hence, it is hard to find the exact value of $N_{g}$. The odd case is harder because of the upper bound $8 g+4$ of $N_{g}$. For the general case we have the following:

Proposition 4.1. Let $f: X \rightarrow \mathbb{S}^{2}$ be a genus-g Lefschetz fibration with $n+s<2 g+4$ and $g>6$. Then the signature of $X, \sigma(X)$, is positive.

Proof. Suppose that $X$ admits a genus- $g$ Lefschetz fibration with $n+s<2 g+4$ for $g>6$ and $g \neq 7$. It follows from Theorem 2.6 that $b_{2}^{+} \neq 1$ and therefore $X$ is not a blow-up of a rational or ruled surface. This gives $c_{1}^{2}(X) \geq 2-2 g$ by [20]. Therefore we get:

$$
\begin{aligned}
2-2 g \leq c_{1}^{2}(X) & =3 \sigma(X)+2 e(X) \\
& =3 \sigma(X)+2(4-4 g+n+s) \\
& \leq 3 \sigma(X)+2(4-4 g+2 g+3) \\
& =3 \sigma(X)+14-4 g,
\end{aligned}
$$

which implies that $\sigma(X)>0$ when $g>6$ and $g \neq 7$.

We see that the same argument holds for $g=7$ using Remark 3.2.

Remark 4.2. Proposition 4.1 implies that every hyperelliptic genus- $g$ Lefschetz fibration with $n+s<2 g+4$ and $g>6$ has $b_{1}(X)>\frac{8 g-15}{6}$ by applying the inequality (2.1). However, the existence of such a Lefschetz fibration is not known [3, 27].

Remark 4.3. Recently, Korkmaz has constructed a factorization of the identity in the hyperelliptic mapping class group $\operatorname{HMod}_{g}$ with length $5 g-3$. This new construction provides us to improve the upper bound of $N_{g}$ when $g$ is odd. Therefore, we conclude that $N_{g} \leq 2 g+4$ if $g$ is even and $N_{g} \leq 5 g-3$ if $g$ is odd.

\section{REFERENCES}

[1] T. Altunöz: Exotic 4-manifolds and hyperelliptic Lefschetz fibrations, Ph.D. thesis, Middle East Technical University, Ankara, Turkey, 2018.

[2] W. Barth, K. Hulek, C. Peters and A. Van de Ven: Compact Complex Surfaces, 2nd edn. Springer, Heidelberg, 2004.

[3] R. I. Baykur: Small symplectic Calabi-Yau surfaces and exotic 4-manifolds via genus-3 pencils, preprint, arXiv:1511.05951.

[4] R. I. Baykur and M. Korkmaz: Small Lefschetz fibrations and exotic 4-manifolds, Math. Ann. 367 (3-4), (2017), 1333-1361.

[5] J. S. Birman and H. Hilden: On the mapping class groups of closed surfaces as covering spaces, Advances in the theory of Riemann surfaces. Ann. Math. Stud. 66 (1971), 81-115.

[6] V. Braungardt, D. Kotschick: Clustering of critical points in Lefschetz fibrations and the symplectic Szpiro Inequality, Trans. Amer. Math. Soc. 355 (8) (2003), 3217-3226.

[7] C. Cadavid: A remarkable set of words in the mapping class group, Ph.D dissertation, Univ. of Texas, Austin, 1998.

[8] E. Dalyan, E. Medetoĝullari, M. Pamuk: A note on the generalized Matsumoto relation, Turk. J. Math. 41(2017), 524-536.

[9] S. K. Donaldson: Lefschetz fibrations in symplectic geometry, Doc. Math. J. DMV. Extra Volume, ICMII (1998), 309-314.

[10] S. K. Donaldson: Lefschetz pencils on symplectic manifolds, J. Differential Geom. 53 (2) (1999), 205-236.

[11] H. Endo: Meyers signature cocycle and hyperelliptic fibrations, Math. Ann. 316 (2000), $237-257$.

[12] R. E. Gompf: The topology of symplectic manifolds, Turkish J. Math. 25 (2001), 43-59.

[13] R. E. Gompf and A. I. Stipsicz: 4-manifolds and Kirby calculus, Graduate Studies in Mathematics, vol. 20, American Math. Society, Providence 1999. 
THE NUMBER OF SINGULAR FIBERS IN HYPERELLIPTIC LEFSCHETZ FIBRATIONS 15

[14] N. Hamada: Upper bounds for the minimal number of singular fibers in a Lefschetz fibration over the torus, Michigan Math. J. 63 (2) (2014), 275-291.

[15] H. Kneser: Die kleinste Bedeckungszahl innerhalb einer Klasse von Flä chenabbildungen, Math. Ann. 103 (1930), 347-358.

[16] M. Korkmaz: Noncomplex smooth 4-manifolds with Lefschetz fibrations, Internat. Math. Res. Notices 2001 (2001) (3), 115-128.

[17] M. Korkmaz: Low-dimensional homology groups of mapping class groups: a survey, Turk. J. Math. 26 (1) (2002), 101-114.

[18] M. Korkmaz and B. Ozbagci: Minimal number of singular fibers in a Lefschetz fibration, Proc. Amer. Math. Soc. 129 (5) (2001), 1545-1549.

[19] M. Korkmaz and A. Stipsicz: Lefschetz fibrations on 4-manifolds, Handbook of Teichmüller theory. Vol. II, IRMA Lect. Math. Theor. Phys., vol. 13, Eur. Math. Soc., Zurich, 2009, pp. 271-296.

[20] T.-J. Li: Symplectic Parshin-Arakelov inequality, Internat.Math. Res. Notices 2000 (18) (2000), 941-954.

[21] T.-J. Li and A. Liu: Symplectic structure on ruled surfaces and a generalized adjunction formula, Math. Res. Lett. 2 (4) (1995), 453-471.

[22] A. Liu: Some new applications of the general wall crossing formula, Math. Res. Lett. 3 (1996), 569-585.

[23] Y. Matsumoto: On 4-manifolds fibered by tori II, Proc. Japan Acad., 59A (1983),100-103.

[24] Y. Matsumoto: Lefschetz fibrations of genus two a topological approach, Proceedings of the 37th Taniguchi Symposium on Topology and Teichmüller Spaces, ed. Sadayoshi Kojima et al., World Scientific (1996), 123-148,

[25] N. Monden: On minimal number of singular fibers in a genus-2 Lefschetz fibration, Tokyo J. Math. 35 (2) (2012), 483-490.

[26] B. Ozbagci: Signatures of Lefschetz fibrations, Pacific J. Math. 202 (1) (2002), 99-118.

[27] I. Smith: Torus fibrations on symplectic four-manifolds, Turk. J. Math. 25 (2001), 69-95.

[28] A. Stipsicz: On the number of vanishing cycles in Lefschetz fibrations, Math. Res. Lett. 6 (3-4) (1999), 449-456.

[29] A. Stipsicz: Singular fibers in Lefschetz fibrations on manifolds with $b_{2}^{+}=1$, Topology Appl. 117 (1) (2002), 9-21.

[30] A. Stipsicz and K.-H. Yun: On minimal number of singular fibers in Lefschetz fibrations over the torus, Proc. Amer. Math. Soc. 145 (8) (2017), 3607-3616.

[31] C. Taubes: $S W=\Longrightarrow G r$ : From the Seiberg-Witten equations to pseudo-holomorphic curves, J. Amer. Math. Soc. 9 (1996), 845-918.

[32] G. Xiao: Surfaces fibrée en courbes de genre deux, Lecture Notes in Mathematics, 1137 Springer-Verlag, Berlin, 1985.

Department of Mathematics, Middle East Technical University, Ankara, Turkey

E-mail address: atulin@metu.edu.tr 